\title{
Enfoque geográfico de los problemas ambientales con énfasis en Colombia: uso de los SIG en el manejo de ellos
}

\section{Oscar Buitrago Bermúdez*}

Resumen. Al pensar en cuál debe ser un enfoque geográfico de los problemas ambientales en Colombia y de qué manera el uso de los Sistemas de Información Geográfico (SIG) aporta en el manejo de ellos, lleva a buscar en primer lugar, un marco de conceptos que de una u otra manera permitan perfilar las posibles respues tas a las preguntas mencionadas. Dicho marco, estaría principalmente conformado por tres elementos que a lo largo de este ensayo se pretenden integrar: de un lado, están las tendencias teóricas que maneja la geografía contemporánea a la cual, por ser una ciencia interesada en los fenómenos socioespaciales, se le pueden reclamar aportes al manejo de la cuestión ambiental; de otro, el concepto de problema ambien tal en un mundo en plena globalización, con una economía de mercado que lanza a la sociedad al consumo y en donde cualquier fenómeno que ocurra en un lugar del planeta, repercute en lugares tan extremos como nunca antes había ocurrido, y desde luego, es en este punto en el que se hace énfasis en el caso colombiano; y por último, las herramientas con que cuentan las sociedades científicas y las personas encargadas de la gestión, ya sea del ambiente o del territorio, para la generación en tiempo real de modelos más acertados de la realidad, capaces de predecir el comportamiento de fenó menos naturales y su relación con el subsistema social, y que además, faciliten la interactividad entre los diferentes núcleos académicos y dirigentes.

(Aceptado noviembre 2002).

\footnotetext{
* Ingeniero Agrícola, Universidad nacional. Magister en Geografía énfasis en ordenamiento territorial, EPG, Convenio UPTC-IGAC, Bogotá. Catedrático universitario y consultor.
} 


\section{Enfoques geográficos de la relación ser humano-naturaleza}

El actual nivel de conocimiento de la superficie terrestre es el resultado de un proceso histórico que ha pasado desde la simple descripción de sus componentes, hasta conceptos más complejos como los sistemas; pero de cualquier manera, es un trabajo de muchos siglos y de generaciones que han propendido por un mejor entendimiento de la realidad humana. Lo anterior justifica presentar una pequeña síntesis de los enfoques geográficos del medio ambiente.

Como ciencia, puede decirse que la geografía tiene sus orígenes en la Grecia del siglo V a.C. En aquella época la geografía conformaba una unidad con la filosofía y la historia y tenía por objetivo dar una explicación científica al entorno y al mundo conocido. A partir de esta época y hasta los grandes descubrimientos realizados por la civilización europea del siglo $\mathrm{XV}$, la geografía presenta un enfoque descriptivo del entorno. Sin embargo, este hito histórico demanda un replanteamiento de la forma de ver el mundo: el planeta es un espacio geográfico en proceso de integración, y la geografía debe ponerse a tono con la situación, por lo que orienta sus objetivos al conocimiento preciso de los nuevos continentes, proceso en el que la cartografía se convierte en una disciplina de fundamental apoyo a la geo- grafía, y mutuamente inician un proceso de crecimiento (Mateo, 2000; Ortega, 2000).

Al llegar los siglos XVIII y XIX, la geografía se encuentra con grandes transformaciones sociales que la obligan a replantear sus objetivos para ponerse a tono con la realidad del momento. La revolución industrial y las confrontaciones de los núcleos de poder europeo por el dominio de los nuevos territorios, hacen que la simple descripción de la superficie terrestre no sea suficiente para las nuevas necesidades, y se presenta una adición importante a esa sencilla abstracción: la explicación del entorno. Este paso permitió «plantear de nuevo la auténtica naturaleza de los fenómenos geográficos». (Ortega, 2000:113). Es en esta época cuando las disciplinas empiezan a determinar sus límites, y es así como la geografía comienza a definir sus discursos epistemológicos y diseños metodológicos que la hacen diferente de otras ciencias $\mathrm{y}$ que por tanto, le permiten abordar con precisión la explicación del entorno, buscando el establecimiento de las relaciones entre los humanos y la naturaleza (Saushkin, 1980, citado por Mateo, 2000). Desde luego, en este periodo la geografía adquiere cimientos más fuertes cuando se identifica su importancia en la vida práctica y sobre todo, en el conocimiento del territorio nacional como un pilar de la identidad de los nuevos estados (Ortega, 2000). 
Esta pequeña síntesis evidencia que hasta principios del siglo $\mathrm{XX}$ el enfoque geográfico de lo ambiental nació del interés de esta ciencia por comprender en diferentes escalas, la compleja interacción entre la naturaleza Natural y la naturaleza Humana. Durante los últimos dos siglos, para abordar la comprensión de estas relaciones en la geografía, según Ordoñez (1991) citado por Mateo (2000), se identifican tres enfoques principales: el ecológico, el corológico y el espacial.

El enfoque ecológico, propio del siglo XIX, consideró que la geografía como ciencia tenía una gran oportunidad para generar, a partir de argumentos positivistas, modelos explicativos de las relaciones entre la sociedad y la naturaleza, para lo cual se entendió la naturaleza como la base de la sociedad y escenario vital para su desarrollo. Este enfoque acuñó el término de medio geográfico que fue entendido como el conjunto de factores y elementos físicos que conforman un área determinada y por tanto, son condicionantes geográficas para el grupo social que la habita (Ortega, 2000). Una de las tantas derivaciones de este enfoque y que actualmente está en fuerte vigencia es el enfoque holístico (integrador), en el cual se acepta la complejidad de los fenómenos naturales y sociales y se desarrolla la noción de sistema geográfico o geosistema, base fundamental de las consideraciones teóricas de este ensayo, por lo que más adelante se volverá sobre él.
El segundo enfoque de las relaciones naturaleza-ser humano, según Ortega (2000) nacido del concepto de medio geográfico de la tradición positivista, define un concepto de mayor trascendencia y significado en la geografía, el de región. Este concepto permite enfocar la observación de una determinada área hacia elementos comunes que singularizan el espacio geográfico y por varias décadas fue el pilar del método inductivo en la geografía regional. Actualmente, este concepto se está recuperando en la medida en que a partir su acepción empirista, permite comprender y explicar diferencias y similitudes de procesos, formas y grados de evolución de los fenómenos socioespaciales a diferentes escalas y en marcos territoriales configurados por diferentes tipos de poderes sociales, entre ellos el Estado. En este sentido, la región surge en determinadas condiciones, se expande, mantiene o desaparece en la medida en que sus factores formadores así lo hagan. Este enfoque se ha cruzado en bastantes puntos con otro concepto clave de la geografía moderna: el paisaje, especialmente cuando se habla de paisaje cultural (Mateo, 2000), el cual aporta mayores elementos para comprender el entorno, conceptualizándolo como una combinatoria de múltiples elementos, físicos y humanos, que son el resultado de un proceso histórico en un área determinada (Ortega, 2000).

El último, es el enfoque espacial o de las regularidades espaciales que pue- 
den ser elevadas al rango de leyes espaciales, con lo cual se continuó en la línea positivista. Este enfoque hace de la distribución de los fenómenos sociales el núcleo de la geografía, dando así un paso adelante al poner en el ámbito geográfico el concepto de organización del espacio, lo que hace que el concepto de espacio se convierta en el centro de la geografía a cambio de los de medio geográfico y región. En este marco conceptual, la actividad humana pasa a jugar un mayor papel que la base natural, estableciéndose que dicha actividad crea estructuras espaciales que definen los procesos geográficos (Unwin, 1993).

Esta corta síntesis de los enfoques geográficos para el abordaje de las relaciones entre el ser humano y la naturaleza, permite decir que en la ciencia geográfica se han propuesto y usado diversas formas de interpretar el mundo, unas veces haciendo fuertes simplificaciones y otras parcelizando la realidad; de cualquier manera, en su memento histórico estas propuestas ideológicas respondieron a unas necesidades específicas y propias de él.

La geografía de hoy se enfrenta a una realidad que cada día adquiere mayores matices y se reconoce más compleja, por lo que no debe ser parcelizada y por el contrario, debe ser entendida como una realidad multidimensional. El actual estado de la ciencia, resultado de un devenir histórico en el que se dieron ideas antagonistas y que ahora más bien pueden ser entendidas como complementarias, sin dejar de ser antagonistas, se presenta con las condiciones de madurez suficientes para abordar sin temores el estudio de la complejidad y aceptar que en ese proceso existen la fuerza de la incertidumbre y la ambigüedad (Morin, 1998).

\section{Los sistemas y la geografía}

Para poder afrontar este nuevo reto, el pensamiento de la humanidad ha propuesto que toda organización se comporta como un sistema, el cual se entiende como un todo que está compuesto por diferentes elementos ensamblados y articulados entre sí; de este modo, el sistema es mucho más que la suma de las partes. Pensadores contemporáneos han llegado a concluir que existen leyes generales de sistemas aplicables a cualquier sistema de determinado tipo, sin importar las propiedades particulares de éste, ni los elementos particulares; dichos pensamientos se concretan en la teoría general de sistemas, la cual se interesa en los problemas de relaciones, de estructuras y de interdependencia entre elementos, más que en la definición de los atributos constantes de ellos. Las relaciones entre los elementos de un sistema pueden ser de tipo lineal, paralelas de retroalimentación, combinadas simples o combinadas complejas, las cuales se pueden medir en términos de flujos, estímulos y respuestas. Los sistemas pueden ser abiertos, cerrados, simples estructuras o sistemas trascendentales (Odum, 1986). 
En la geografía, de acuerdo a Unwin (1993), desde principios de la década de los setenta se introduce la teoría de Sistemas que había sido desarrollada por Bertalanffy hacia mediados del siglo XX. Los geógrafos que apoyaban el uso de esta teoría consideraban que aportaba la estructura conceptual "para el análisis que permite combinar los fenómenos humanos y los ambientales" (Unwin 1993:180).

Según Stoddart, citado por Unwin (1993), manejar el concepto de ecosistema en geografía trae las siguientes ventajas: agrupa elementos humanos, biológicos y zoológicos, en una estructura única e integrada, su estructura facilita el estudio lógico del espacio y por último, se concibe la realidad a partir de flujos continuos de entradas y salidas. Al igual que Stoddar, Tricart (1989:159-176) considera que "un enfoque sistémico puede ser usado para integrar los múltiples resultados analíticos obtenidos por varias disciplinas tradicionales y para investigar el flujo de retro-alimentación entre los fenómenos". Brunet y Dolfus (1991) consideran que para conocer el funcionamiento del espacio y más aún, para encontrar la diferenciación espacial (labor del geógrafo) no es suficiente con la descripción de elementos y acciones, sino que es necesario establecer claramente las relaciones, las cuales a su vez tienen tarde o temprano una expresión espacial, hecho que lleva a pensar en un sistema o en sistemas de elementos (objetos) y acciones (Santos, 2000) como componentes del espacio geográfico.

La geografía ha propuesto la utilización del concepto de geosistema que corresponde a un determinado tipo de sistema (De Bolos, 1992). Este concepto permite abordar desde la complejidad, la interpretación multidimensional y la construcción de modelos teóricos del espacio geográfico. Más parado en la geografía física Bertrand citado por Scheibling (1994) propone al geosistema como su concepto central por su origen naturalista, espacial y temporal y además, por que cuenta con una dimensión antrópica y por ser capaz de integrar todas sus subdisciplinas. Se puede concluir que estas posiciones conceptuales buscan tener en cuenta la interactividad de los fenómenos naturales y humanos a cualquier escala.

De acuerdo con De Bolos (1992), existen en el geosistema diversos tipos de elementos fundamentales que pueden ser considerados subsistemas de primer y segundo orden, entre los que destaca:

El subsistema biótico, que comprende a su vez subsistemas no dotados de vida y que por su condición de poca variabilidad temporal, son altamente estructurantes de los geosistemas. A este subsistema corresponden el basamento litológico, el agua y el aire, por ejemplo. 
- El subsistema biótico, que considera a su vez subsistemas con vida (flora, fauna, ser humano) y que presenta con los demás, relaciones similares por ser parte de las cadenas tróficas. Este subsistema es lo que en ecología de llama ecosistema. Se pueden considerar como bioindicadores del geosistema.

- El subsistema organizado por el ser humano o antrópico, que expresa las construcciones hechas por el ser humano para vivir en sociedad y mantener un modo económico que le satis- faga sus necesidades materiales y espirituales.

Entre estos subsistemas se presentan otros de transición: por ejemplo entre el subsistema abiótico litológico y el subsistema biótico vegetal, se presenta una interface que se denomina el subsistema edáfico. El geosistema, al igual que los demás sistemas terrestres se considera abierto, es decir que en él entra o sale cierta cantidad de materia, de energía y de información haciéndolos unos sistemas dinámicos.

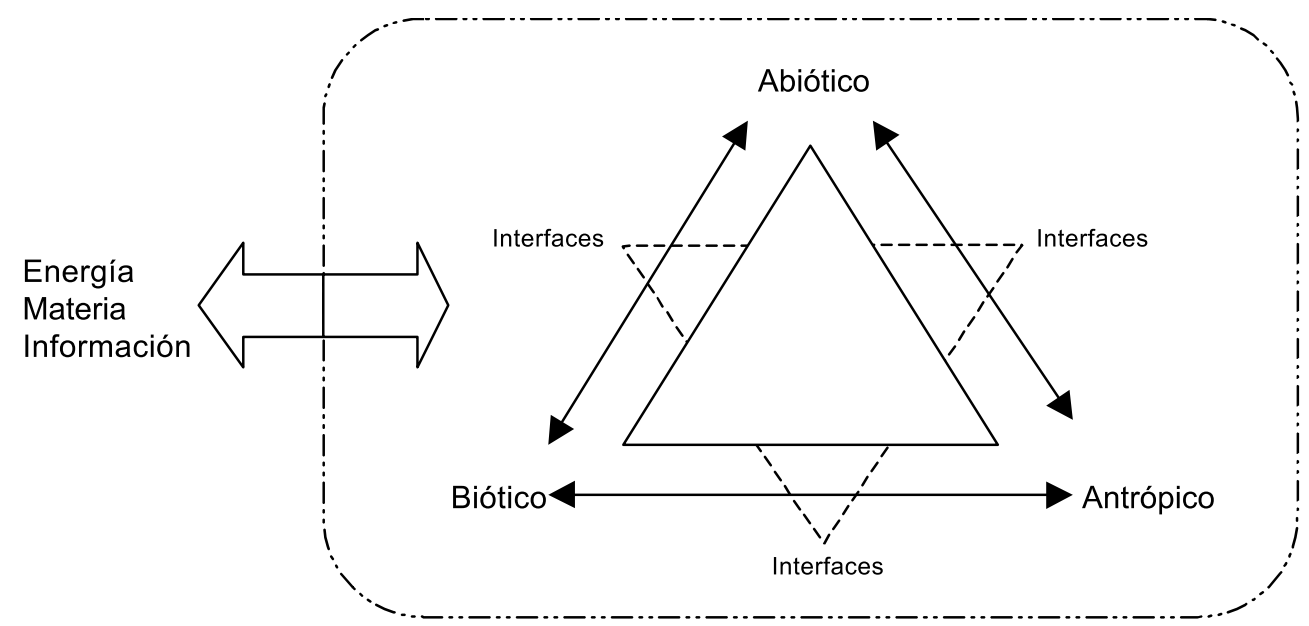

Figura 1. Representación de un geosistema a partir de los subsistemas propuestos por De Bolos, 1998.

\section{Enfoques conceptuales integradores}

Una primera integración de dos enfoques geográficos en el abordaje de las relaciones entre los seres humanos y la naturaleza se da con la aplicación del enfoque sistémico al concepto de región. Aquí se concibe y conceptúa a la región como un sistema regulado por los flujos materiales (bienes, per-

126 Oscar Buitrago Bermúdez 
sonas, mercancías), e inmateriales (servicios, información), dentro de los mismos límites regionales y con el exterior, según los postulados de Brunet, citado por Ortega (2000). El mismo autor dice que el geosistema región «evoluciona de acuerdo a las condiciones internas y externas, a las influencias recíprocas, en que intervienen tanto componentes físicos como sociales» (Ortega, 2000: 483).

Otro enfoque integrador de conceptos bastante válido para interpretar la complejidad de la realidad, es el propuesto por De Bolos (1992), quien aplica la teoría de sistemas para abordar el estudio del paisaje. En este sentido, ella establece que el paisaje se conceptualiza como un sistema, queriendo decir que el geosistema es el modelo de aquel.

La formulación de un marco teórico basado en el concepto de geosistema permite identificar los niveles de coherencia en una región o un paisaje, es decir cuáles de sus componentes y relaciones están en armonía y cuáles no (Cáncer, 1999).

\section{La degradación del paisaje es problema ambiental}

Para comprender la degradación del paisaje como un problema ambiental es necesario considerar dos conceptos: por un lado, el concepto de deterioro y, por otro, el de problema ambiental.
El primero se entiende como la ausencia de armonía en un paisaje o lo que es igual, según Puyol et al, 1986, citado por Cáncer, 1999, su empobrecimiento cuantitativo y cualitativo como resultado de fenómenos antrópicos o naturales. Este concepto está basado en consideraciones de carácter estético, puesto que en él se habla de la armonía como un precepto determinante en la definición del deterioro del paisaje, lo que lleva a concluir que el concepto de deterioro de un paisaje es perceptual y por tanto, depende de quien lo observa.

De otra parte, el concepto de problema ambiental desde la perspectiva ecológica está determinado por el individuo, la cual en términos generales establece que un problema ambiental es todo lo que se oponga a los objetivos de la gente (Carrizosa, 1992). Así, el problema ambiental es definido en términos de percepción, valoración y actitud del sujeto. De acuerdo a lo que el individuo percibe como problema ambiental, éste definirá una escala de valoración y por tanto una actitud frente al problema. La escala de valoración poseerá un umbral crítico, el cual al ser sobrepasado generará condiciones problemáticas. Nótese que la escala de valores y el umbral son relativas al sujeto. En general la escala de valores y el umbral, corresponden a diferentes dimensiones de acuerdo a las preguntas lógicas fundamentales sobre el espacio, el tiempo, la sociedad, la técnica, la economía y la cultura: dónde, cuán- 
do, para qué, cómo, cuánto y para quién, enmarcan el proceso de decisiones y hacen percibir referentes de umbrales en los sistemas; así por ejemplo, la utilización de suelos agrícolas para actividades de industrialización, traspasa un umbral crítico sí este cambio de uso se percibe como un problema ambiental, lo cual se puede dar en aquellos países donde la escasez del suelo se considera un peligro para la supervivencia de las generaciones futuras (Ortega y Rodríguez, 1997).

Como se ve, el punto de encuentro entre lo que se entiende por degradación del paisaje y problema ambiental, está en la base subjetiva de ambos conceptos, lo cual lleva a pensar que una disciplina como geografía, interesada en el paisaje y en la región, debe formularse objetivos de carácter ético-social y educativo-formativo en la búsqueda de la protección integral del ambiente.

\section{La problemática ambiental en Colombia}

Los enfoque geográficos antes mencionados aportan los siguientes elementos para abordar la interpretación de la problemática ambiental en Colombia, desde las perspectivas académica y administrativa del Estado:

- La comprensión de que el ser humano y su entorno conforman una unidad que funciona sistemicamente, es decir, que sus componentes son complejamente interactivos y por tanto, de una $u$ otra manera, el cambio de alguno de ellos provoca efectos sobre todo el geosistema.

- Los atributos espaciales de los geosistemas, permiten la identificación de patrones espaciales, así como la identificación de diferencias y similitudes con otros fenómenos parecidos, ocurridos en otros lugares del planeta, a partir de lo cual se pueden identificar soluciones parecidas, a problemas locales.

- El componente ético-social del geosistema, comprendido como la construcción mental y social de escalas de valoración del significado e importancia de un cambio en el geosistema, hace parte de la dimensión antrópica del mismo. Por lo anterior, la comprensión del funcionamiento de un geosistema debe considerarse en el sentido más amplio, la percepción humana y su relación con la ética social.

a Como resultado de los dos elementos anteriores, se considera que la problemática ambiental colombiana debe ser abordada, por la misma complejidad que ella comprende, con perspectiva interdisciplinar.

En la figura 2, se representa un modelo conceptual del abordaje de la problemática ambiental en Colombia desde la perspectiva geográfica hasta ahora estudiada. 


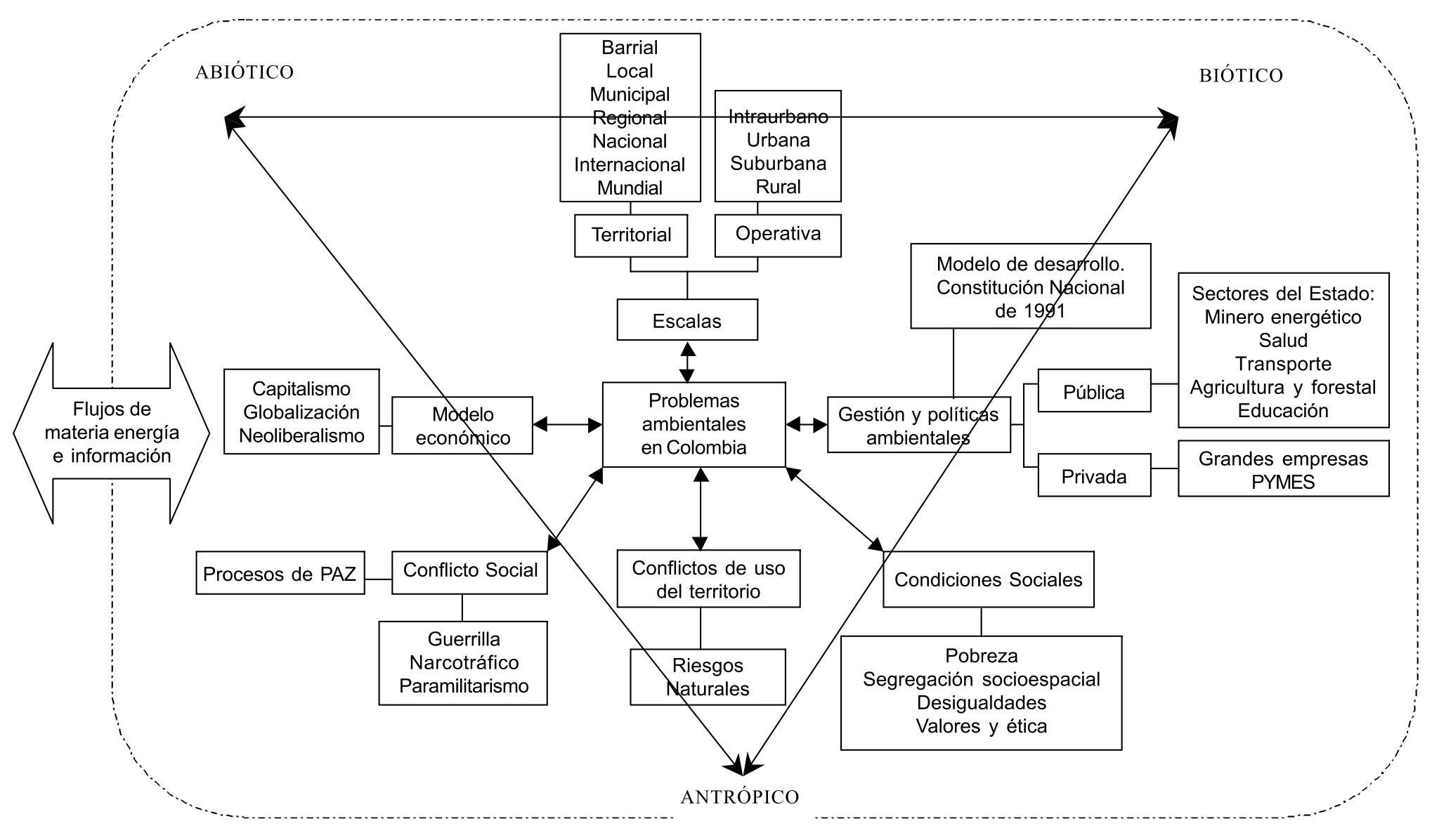

Figura 2. Expresión sintética de la complejidad de los problemas ambientales en geosistema Colombia. 
El modelo parte de considerar que todos los componentes del geosistema colombiano (biótico, abiótico y antrópico) interactúan entre sí y, este a su vez, interactúa con el geosistema planetario. Este geosistema en su actual fase histórica, presenta problemas ambientales propios de este momento, pero que son el resultado de procesos antiguos tanto en el tiempo geológico como en el tiempo histórico humano.

Al considerar la problemática ambiental desde la perspectiva de la complejidad y la sistémica, se facilita establecer derroteros de enfoques para el abordaje geográfico de los mismos. Así por ejemplo, derroteros derivados del modelo y que pueden ser concebidos como un problema investigativo, podrían ser:

$\square$ ¿Cuáles son los problemas ambientales generados por el sector industrial en el barrio Aguas Claras de la localidad de Engativá en Bogotá?

¿ ¿Cuáles son los problemas ambientales derivados de la globalización en las ciudades andinas colombianas? ¿En qué puntos se diferencian y por qué?

$\square$ ¿Qué factores explican el interés de los grupos insurgentes por los territorios del pie de monte llanero? ¿Cómo dichos factores determinan los problemas ambientales en la región?

La formulación de estas pocas preguntas, a partir del modelo conceptual in- tegral evita poner de lado el resto de los componentes del geosistema.

Considerando la complejidad de la actual problemática ambiental en Colombia como se deduce del modelo expuesto y a manera de ejemplo, se exponen algunos aspectos de la relación existente entre problemas ambientales y conflicto social a escala nacional, basado en Reyes (1998) y Reyes (1999), como uno de los tantos derroteros derivados del mismo modelo, para después culminar con el último aspecto del ensayo.

\section{Conflicto actual en Colombia y su incidencia ambiental}

La ausencia o casi nula presencia del Estado en muchas regiones del país, propicia profundos desequilibrios en el desarrollo del territorio nacional. En general, se observa una red de ciudades altamente articuladas, pero que dejan espacios totalmente aislados y a expensas de fuerzas ilegítimas, como lo son la guerrilla y el paramilitarismo, quienes en cierta medida han suplido las insuficiencias del Estado, por ejemplo, garantizando protección contra las acciones guerrilleras o la delincuencia común, protección de los recursos naturales o abanderando ideales de igualdad social.

Este fenómeno denominado ingobernabilidad, propicia una serie de conflictos territoriales que se pueden categorizar en dos ordenes: los rura- 


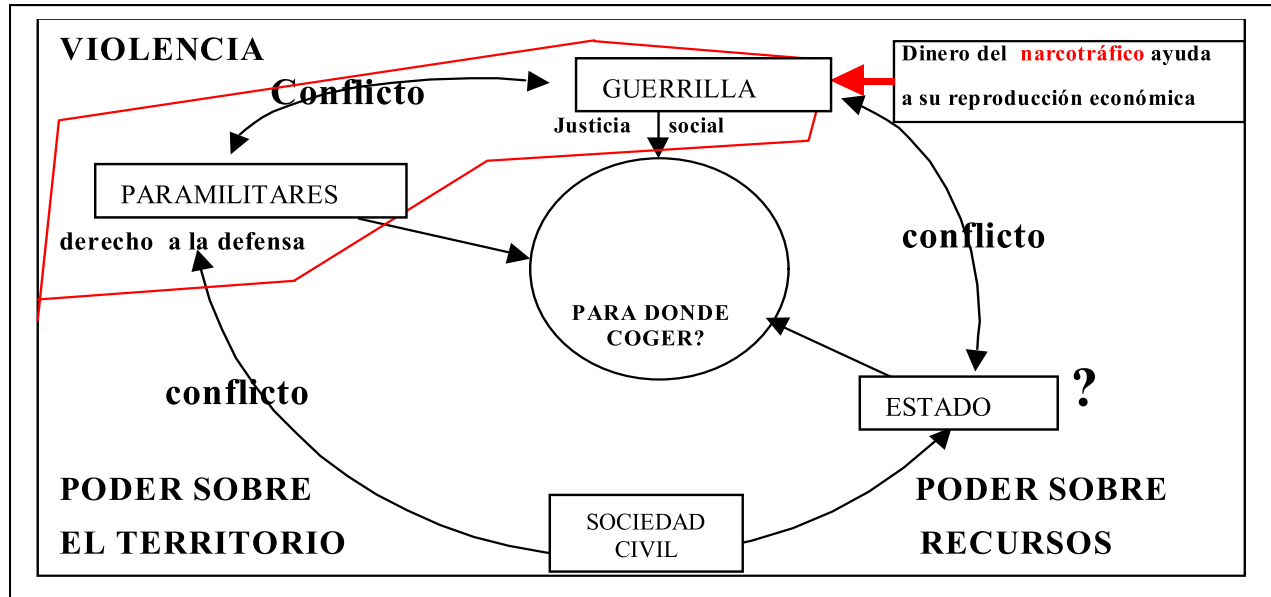

les y los urbanos. En los espacios de operación rural se encuentran dos actores principales: por un lado, la guerrilla reclamando justicia social al Estado y ampliando las fronteras de su gran imperio de extorsión y terror, y por otro, los paramilitares, quienes con sus acciones consideran que están protegiendo a la gente de bien de las acciones guerrilleras, por cual cobran un tributo con el que toman vigor para continuar su cometido.

En la ciudad, están las acciones violentas que tienen que ver directamente con la crisis que sufre el campo, mencionada anteriormente. Las ciudades reciben la población desplazada del campo que va a refugiarse a las periferias de los espacios urbanos en donde las carencias y necesidades en sus máximas expresiones, se conjugan con asentamientos en zonas de alto riesgo natural, propiciando nichos de inconformidad que son aprovechados por la guerrilla para ampliar su espacio de acción; de igual manera, en la ciudad están los grupos de limpieza privada y la mano de obra criminal al servicio del narcotráfico.

\section{El conflicto socioambiental}

Disputando el dominio del territorio nacional se encuentran cuatro actores principales que propician conflictos socioambientales en su interacción: por un lado, están la guerrilla, los paramilitares y los narcotraficantes y por el otro, el Estado en su condición de dominio legal sobre el mismo.

\section{Narcotráfico y los problemas ambien- tales}

La presencia de sistemas productivos ilícitos en el territorio nacional ha permitido la conformación de unos actores sociales, los narcotraficantes, quie- 
nes disputan el territorio con miras a garantizar el funcionamiento de sus negocios. Su poder se encuentra concentrado en los valles de los ríos Magdalena y Cauca y en la Orinoquia, siendo su principal actuación espacial la compra de tierras altamente productivas y el fomento de cultivos ilícitos en zonas de colonización.

La compra de tierras altamente productivas y su destinación a sistemas ganaderos extensivos, resta posibilidades a la producción agrícola del país y por tanto, pone en riesgo el aseguramiento alimentario de la población colombiana. Lo anterior, aunado al proceso continuo de compra de tierras, hace que este actor sea determinante en la ordenación de los territorios rurales. Con estas actividades usurpa el liderazgo local de otros sectores de la sociedad. Este grupo social fomenta la violencia al contratar la vigilancia privada que ofrecen los paramilitares.

De igual manera, la producción de sustancias sicoactivas en el territorio nacional trae consigo dos problemas de carácter ambiental. Por un lado, durante el procesamiento se manejan sustancias residuales que contaminan suelos y ríos, ampliando su impacto espacial; de otro, esta el andamiaje montado por el Estado, con el auspicio de Estados Unidos, para la erradicación mediante la fumigación aérea de grandes extensiones, método de aplicación de agroquímicos que pone en el aire grandes cantidades de con- taminantes que son arrastrados por deriva hacia otros lugares, aumentando las áreas impactadas por estos procesos de control.

\section{La guerrilla y los problemas ambien- tales}

La guerrilla es un actor social que apareció en la historia nacional hace varias décadas, cuando la insurgencia se fundamentaba en la lucha por el poder para el mejoramiento de la calidad de vida de las clases sociales menos favorecidas. En la actualidad, se encuentran varios grupos guerrilleros que ejercen su dominio sobre distintas áreas del territorio nacional, en donde para subsistir obligan a tributar a los productores, comerciantes y transportadores.

Las principales regiones afectadas por las acciones guerrilleras son, en orden de importancia el Magdalena Medio, que incluye al sur de Bolívar y Cesar, parte de Santander, Antioquía, Caldas y Boyacá, así como la región del Catatumbo; la segunda es la región comprendida por Córdoba, norte de Chocó, y el noroccidente de Antioquia; la tercera es el norte de Cesar y Magdalena, y el sur de la Guajira, específicamente en los alrededores de la Sierra Nevada de Santa Marta; la cuarta es Arauca y el occidente de Casanare, coincidiendo con una de las cuencas sedimentarias de mayor producción petrolera del país. La guerrilla ha actuado violentamente en cerca del $90 \%$ de los municipios 
del país, atacando principalmente oleoductos y gasoductos, con lo cual agreden y desarmonizan fuertemente el equilibrio de los geosistemas colombianos. En algunas regiones conviven pacíficamente con los habitantes y procuran no actuar militarmente.

La guerrilla se posiciona en el territorio argumentando sus viejos ideales: ser abanderados de los intereses populares y protectores de los débiles y desamparados, hecho que se potencia con la evidente ausencia del Estado y la marcada pobreza de la mayoría de la población. La tributación, el secuestro y la extorsión, son las fuentes de financiación de su guerra; en algunas regiones se han aliado con el narcotráfico para controlar las rutas de comercio de insumos y productos narcóticos.

\section{El paramilitarismo}

Este actor social nace cuando el Estado no es capaz de controlar las acciones armadas de la guerrilla. En un principio fueron auspiciadas por la fuerza militar del Estado, pero con la pérdida de este respaldo, el narcotráfico y los grandes terratenientes entran a apoyar su actividad de guerra contrainsurgente. Ofrecen el servicio de protección privada contra las acciones armadas de la guerrilla, con la cual disputan las rutas de mercadeo de insumos y narcóticos. Además de lo anterior, se encargan de amedrentar y sacar de las regiones que dominan a campesinos, con lo cual facilitan la concentración de la propiedad en unas manos, pero a su vez ponen en riesgo la seguridad alimentaria del país.

Actúan principalmente en el departamento de Córdoba y la región de Urabá, en las zonas esmeraldíferas y, recientemente han ampliado su territorio a departamentos de la Costa Atlántica, Magdalena Medio, Cundinamarca, Casanare, Arauca, Meta, Guaviare, Caquetá y Putumayo.

\section{¿El por qué de esta situación?}

Existe una asociación directa entre la ubicación geográfica de los grupos guerrilleros y paramilitares y la estructura biótica y abiótica del geosistema nacional; estos actores del conflicto se localizan en las zonas de mayores riquezas naturales (mejores suelos, petróleo, agroindustria, etc.). Lo anterior tiene que ver con la posibilidad de mayores ingresos por extorsión, boleteos y vacunas que los grupos económicos, entre otros, deben pagar para poder desarrollar su actividad. Estas zonas se convierten en espacios de excelente reproducción económica para guerrilleros y paramilitares, contrario a lo que sucede en las regiones pobres del país, las cuales por sus mismas condiciones de pobreza e insatisfacción, se convierten en espacios apropiados para la reproducción social de estos grupos. En últimas, el Estado se reduce a las formales elecciones de gobernadores y legisladores, lo cual aunado a sus vicios 
(corrupción, deshonestidad y favoritismo) aumenta las probabilidades de guerra.

En conclusión, el abordaje desde la perspectiva geográfica de este problema socioambiental involucra elementos estructurales del subsistema antrópico que tienen que ver por ejemplo, con la administración que el Estado le da a los recursos naturales, la intervención internacional en conflictos socioespaciales internos o la mundialización de los mercados de narcóticos, entre otros muchos elementos a considerar.

\section{Los sistemas de información geográfica y los geosistemas}

Hablar de Sistemas de Información Geográfica (SIG) y de geosistemas, evidencia muchos puntos de encuentro, nada más empezando por sus nombres. Sin embargo, la congruencia mayor está en que ambos enfoques ofrecen la posibilidad de crear modelos de la realidad: los SIG a través de aplicaciones computacionales, y los geosistemas, como ya se vio, a través de la determinación de estructuras de la realidad.

Adicional a lo anterior, es necesario indicar que el actual estado del proceso de globalización, caracterizado por el alto desarrollo de las tecnologías de comunicación y manejo de la información, es el marco en cual se conciben los sistemas de información geográfi- ca, más haciendo parte de él o quizás como un resultado, que teniéndolo como un referente.

El disponer de tecnologías computacionales que permitan modelar la realidad, facilita la integración a los niveles deseados por el usuario, de las variables físicas y humanas de la base de datos del SIG, situación que amplía de lleno, las posibilidades de evaluación de la realidad, sin estar inscrito en una determinada escuela epistemológica (Buzai, 2000).

\section{Los SIG: tecnología para el mejor conocimiento $y$ manejo de los problemas ambientales}

La adopción de la tecnología SIG como herramienta que permite modelar la realidad, facilita ante todo realizar tres operaciones importantes: primero, conocerla como un sistema integral de variables bióticas, abióticas y antrópicas, a partir de lo cual se puede identificar el estado de evolución del paisaje, ya sea éste artificial, natural o en proceso de transformación; planificar las acciones necesarias para reorientar la evolución del paisaje; y por último, controlar y evaluar los procesos, tanto naturales como humanos, que componen el geosistema (De Mers, 1997; Clarke, 1997).

Esto lleva a pensar que un proceso de gestión y planificación ambiental requiere de la recopilación y generación 
de grandes cantidades de información relacionadas con subsistemas bióticos, abióticos y antrópicos y con las actividades administrativas y de funcionamiento del medio ambiente. Desde luego, que la manipulación y manejo de la información debe estar orientada a la producción de modelos de la realidad que ante todo sean el fundamento de la toma de decisiones para la gestión de los procesos de planificación ambiental, por lo que se puede asegurar que el mejor camino para lograr este propósito es mediante la implementación de un Sistema de Información Geográfica.

De otra parte, los SIG al ser compatibles con otras tecnologías de captura de información como los sensores remotos, mejoran el conocimiento de la realidad y por lo tanto, facilitan los procesos de toma de decisiones, haciendo que éstas se puedan llevar a cabo casi en tiempo real, especialmente cuando se trata de eventos desastrosos, sean estos de orden natural o antrópico.

En estas condiciones, la tecnología SIG en los procesos de planificación ambiental, es capaz de responder a las necesidades que tienen los encargados de la gestión de hacer diagnóstico, evaluación y seguimiento, de manera eficiente, eficaz, ágil, oportuna (en tiempo real) y precisa, a todas aquellas actividades humanas que de una $u$ otra manera, intervienen el medio ambiente, lo cual ante todo es posible si se maneja como criterio fundamental el que la calidad y pertinencia de la información es el soporte para realizar acertadamente los procesos de planeación y toma de decisiones.

Con los SIG los actores del sector ambiental pueden obtener información precisa y suficiente que les permita planear, priorizar, orientar y canalizar la inversión de los recursos, tanto humanos como económicos, en la formulación y desarrollo de programas y proyectos ambientales. De otra parte, el carácter dinámico y permanente de los SIG, permite actualizar continuamente la información, de tal manera que se facilita la detección y el registro de la evolución de características y magnitudes de los hechos geográfico-ambientales.

En el caso tratado, modelar la realidad de los actores sociales del conflicto, mediante la tecnología SIG, considerando a las diferentes regiones del país como geosistemas en los que se identifiquen las estructuras principales y los elementos en disputa (biótica, abiótica y antrópica), haciendo énfasis en las relaciones que se establecen entre los componentes, ya sea para el establecimiento del dominio en el territorio por alguno de los actores o para la reproducción social o del capital y en los efectos socioambientales que este tipo de relaciones conlleva, aportaría elementos de juicio, para comprender el estado de deterioro del geosistema en Colombia, y herramientas para la formulación integral de estrategias de paz. 


\section{Conclusiones}

Los diversos enfoques geográficos que han existido para abordar el estudio de las relaciones entre los seres humanos y la naturaleza, han hecho que se llegue a reconocer la complejidad de ellas, pero a la vez, han propiciado la formulación de nuevas formas de pensamiento que sin temor aborden este reto, esto es el pensamiento sistémico. De igual manera, los enfoques han llevado a reconocer que el ser humano y sus actividades son un componente más de los geosistemas y que la concientización de la valoración subjetiva que él hace del estado del paisaje, hará que asuma nuevos valores y propenda por una ética social enmarcada en la protección del ambiente.

Este enfoque, aunado a las posibilidades que ofrecen los SIG para modelar la realidad y simular fenómenos geográficos, permitirá adelantarse virtual- mente a situaciones futuras, por ejemplo en los fenómenos de orden climático o de amenazas naturales, o modificar la trayectoria histórica de los mismos, caso del crecimiento de ciudades, la deforestación, entre otros aspectos.

Vistas las bondades de los enfoques geográficos actuales y de la aplicación de la tecnología SIG, en abordaje de los problemas ambientales en Colombia, se puede concluir que nuestro país cuenta con opciones conceptuales y tecnológicas, para conocer mejor su compleja problemática socioambiental y propender por el mayor entendimiento del funcionamiento de sus geosistemas, lo cual facilita y orienta la toma de decisiones hacia el modelo de desarrollo sostenible propuesto en la Constitución Nacional de 1991, hecho que a su vez repercutirá en el mejoramiento de la calidad vida de los colombianos. 


\section{Bibliografía}

ARONOFF, S. Geographic Information Systems: A Management Perspective. Ottawa: WDL Publications, 1989.

BOISIER, Sergio. La geografía de la globalización. Un único espacio y múltiples territorios. CLAD Home page, 1997.

BOSQUE, S. J. Sistemas de Información Geográfica. Madrid: Ed. Rialp S. A. 1992.

BRADFORD, M. y Kent A. Understanding Human Geography. Oxford: Oxford University Press, 1995.

BRUNET y DOLLFUS. Mondes nouveaux, geographie universelle. Traducción de Florez, A, Lecturas de Geografía II, Universidad Nacional de Colombia, Bogotá, 1998.

BURROUGH, P. A.. Principles of Geographical Information Systems for Land Resource Assesment. Oxford, Clarendon, 1989.

BUZAI, D. Gustavo. La exploración geodigital. Buenos Aires: Lugar Editorial, 2000.

Geografía Global. Buenos Aires: Lugar Editorial, 2000.

CARRIZOSA, U. Julio. La política ambiental en Colombia. Desarrollo sostenible y democratización. Bogotá: FESCOL, CEREC, FONDO FEN COLOMBIA, 1992.

CANCER, L. A. La degradación y la protección del paisaje. Madrid: Ediciones Cátedra S. A. 1997.

DE BOLOS, María. Manual de ciencia del paisaje. Teoría, métodos y aplicaciones. Barcelona: Masson, Colección de Geografía, 1992.

DE MERS M. Fundamentals of Geographic Information Systems. John Wiley \& Sons, Inc. New York, 1997.

INSTITUTO GEOGRÁFICO AGUSTIN CODAZZI. Conceptos Básicos sobre Sistemas de Información Geográfica y Aplicaciones en Latinoamerica. Bogotá: IGAC, 1995.

HAGGET Peter. Geografía: una síntesis moderna. Barcelona: Ediciones Omega, S.A. 1983. 
HOLT J. A. Geografía: historia y conceptos. Barcelona: Vicens Vives, 1989.

CLARKE, C. K. Getting started with Geographic Information Systems. Prentice Hall. New Jersey, 1997.

MATEO R., José Manuel. La geografía como sistema de ciencias en la interacción naturaleza-sociedad. En: Geografía para el tercer milenio. México: INSTITUTO DE GEOGRAFÍA-UNAM, 2001.

MAYA J. y SERRANO D. Fundamentos de SIG. Bogotá: notas de Clase, 1998.

MESA, C., ROJAS, H. y ROJAS, M. Herramientas para la participación en la gestión ambiental. Bogotá: FRIEDRICH EBERTO STIFTUNG, DNP, 2000.

MOLDES J. Tecnología SIG. Madrid: Ed. Rama, 1992.

PEREÑA. J. Introducción Conceptual a los SIG. Casa del Libro. 1992.

ORTEGA D., Ramón y RODRÍGUEZ M., Ignacio. Manual de gestión ambiental. Madrid: Editorial MAPFRE S.A. 1994.

ORTEGA V., José. Los horizontes de la geografía. Teoría de la geografía. Barcelona: Ariel Geográfica, 2000.

REYES P., A. Regionalización de los conflictos agrarios y la violencia política en Colombia. En: Municipios y regiones de Colombia. Bogotá: Fundación Social, 1998.

REYES P., A. 35 años de conflicto. Lecturas Dominicales, periódico El Tiempo. 17 de octubre de 1999.

TRICART, J. Earth Natural System: a condition to the geosphera-biophera program. En: Geookodynamic, Band 10:159-176. Traducción de Florez, A., Lecturas de Geografía II, Universidad Nacional de Colombia, Bogotá, 2001.

SANTOS M. La naturaleza del espacio. Técnica y tiempo. Razón y emoción. Editorial Ariel. Barcelona, 2000.

SCHEIBLING, J. Une ou des geographies? Quést-ce que la geographie? 99-116. Paris, Hachette. Traducción de Florez, A, Lecturas de Geografía II, Universidad Nacional de Colombia, Bogotá, 2002.

UNIVERSIDAD DISTRITAL. Memorias Seminario Internacional SIG, 1998.

UNWIN, T. El Lugar de la geografía. Editorial Cátedra. Madrid, 1994.

138 Oscar Buitrago Bermúdez 


\section{Indicaciones para presentación de artículos para la revista Perspectiva Geográfica}

\section{Formato de presentación}

- Word

- Papel blanco bond, tamaño carta

- Márgenes: Izquierda, superior y derecha: $3 \mathrm{~cm}$. Inferior: $2 \mathrm{~cm}$.

\section{Texto}

- Digite el texto a espacio sencillo, utilice Times New Roman, tamaño 12.

- Título del trabajo: centrado, negrilla, mayúsculas.

- A doble espacio: nombre del autor en minúsculas, negrilla con asterisco como llamado para anotar: profesión, títulos e institución donde trabaja.

- Resumen: escriba la síntesis del trabajo en no más de 100 palabras.

- Palabras clave: identifique las palabras principales del trabajo.

- El resumen y las palabras clave, deben venir en español, inglés y francés.

- Títulos del trabajo, numeración arábiga centrados, mayúsculas fijas.

- Subtítulos: izquierdo, negrilla y minúsculas.

- Siguientes subtítulos: izquierdo, minúsculas para los siguientes aportes utilice literales.

\section{Tablas $y$ figuras}

- Enviarlas en archivos separados especificando el Software, en tamaño carta con las márgenes ya mencionadas.

- Las tablas y figuras deben ser enumeradas correlativamente.

- Las figuras (gráficos, mapas, etc.) deben ser presentados en original, tamaño carta.

\section{Referencias bibliográficas}

- Citas textuales cortas incluidas en el texto principal van entre comillas, con su respectiva referencia (apellidos del autor, año de publicación y página exacta de donde se extrajo la cita).

- Transcriba las citas textuales demasiado largas con indentación izquierda a renglón sencillo, sin comillas y con referencia bibliográfica exacta.

- Las referencias generales a una fuente, identificarlas con el apellido del autor y el año de publicación.

- La lista de referencias bibliográficas deben aparecer alfabéticamente, utilice normas ICONTEC para su presentación. 


\section{Maestría en Geografía Énfasis en Ordenamiento Territorial}

Dirigido a: Geógrafos, licenciados, ingenieros y otros profesionales de la ciencias sociales y naturales que demuestren genuino interés por la investigación geográfica aplicada al ordenamiento territorial.

Modalidad: presencial en horario de 7:00 a 11:00 de la mañana.

Ingreso: anual. Inscripciones: septiembre a noviembre.

Título: Magister en Geografía, énfasis en ordenamiento territorial.

\section{Diplomados}

\section{- EN ORDENAMIENTO TERRITORIAL}

Dirigido a: Funcionarios públicos, consultores y profesionales de las ciencias naturales y sociales, relacionados con la dirección, ejecución, supervisión y/o asesoría de planes de ordenamiento territorial, o interesados en adquirir conocimientos y desarrollar habilidades en el manejo de metodologías aplicables a procesos de OT.

- INSTRUMENTOS DE GESTIÓN URBANA PARA EL ORDENAMIENTO TERRITORIAL.

Dirigido a: Alcaldes, funcionarios públicos, consultores, planificadores, geógrafos, urbanistas, arquitectos e interesados en la temática del Ordenamiento Territorial.

- GEOGRAFIA DE LA SALUD

Dirigido a: Profesionales de la medicina, enfermería, administración hospitalaria, geografía y todos aquellos interesados en conocer la perspectiva geográfica de la medicina y salud. 
Perspectiva Geográfica 141 